\title{
PERÍFRASIS E INDUCTORES NEGATIVOS: UN ANÁLISIS EN TÉRMINOS DE DOMINIOS
}

Resumen. En este trabajo nos ocupamos de las estructuras perifrásticas que contienen negación en su interior, siguiendo el esquema $<$ auxiliar $+n o+$ auxiliado $>$, y concretamente de las condiciones bajo las cuales una perífrasis admite la interposición de este adverbio. Argumentamos, mediante un examen detallado de las perífrasis de gerundio e infinitivo, que la interposición de la negación produce una lectura de evento negativo. Las perífrasis que admiten esta interposición son aquellas cuyas condiciones de uso son compatibles con las propiedades semánticas de los eventos negativos. Específicamente, ya que los eventos negativos son no dinámicos, la interposición de la negación solo se admite en aquellas perífrasis que se pueden combinar con auxiliados no dinámicos.

Palabras clave: negación, perífrasis, material interpuesto, dinamicidad, dominios

\section{PERIPHRASES AND NEGATIVE INDUCTORS: A DOMAIN-BASED ANALYSIS}

Abstract. In this article we deal with periphrastic structures that contain a negative trigger inside, following the scheme <auxiliary $+n o+$ auxiliated $>$; specifically, we discuss the conditions under which a periphrasis allows this adverb to be interposed between the two verbs. We argue, through a detailed analysis of gerund and infinitive periphrases, that negation interposition triggers a negative event reading. The periphrases that allow the interposition are those whose conditions of use are compatible with the semantic properties of negative events. Specifically, as negative events are non dynamic, negation interposition is only allowed in the periphrases that can combine with non dynamic auxiliated predicates.

Keywords: negation, periphrasis, interposed material, dynamicity, domains

\section{Introducción}

Las perífrasis verbales han sido objeto de estudio en numerosos trabajos que se han concentrado sobre todo en intentar establecer una clasificación exhaustiva, describir sus propiedades tempoaspectuales y estudiar la combinatoria entre auxiliares (cf. para el español Roca Pons, 1958; García González, 1992; Squartini, 1998; Fernández de Castro, 1999; Laca, 2002; 
García Fernández, 2006; junto a las descripciones generales pormenorizadas que se encuentran en Gómez Torrego, 1999; Yllera, 1999; RAE y ASALE, 2009: §28). Sin embargo, existe un aspecto de su estudio que hasta donde se nos alcanza ha pasado prácticamente desapercibido en la bibliografía: la posibilidad de que aparezca material interpuesto entre el verbo auxiliar y el verbo auxiliado.

El comportamiento de las perífrasis a este respecto no es uniforme. Así, dos perífrasis distintas, tanto en lo que respecta a la forma del auxiliar como a su semántica, no admiten el mismo material interpuesto, como ilustra el contraste de (1). En (1a), donde tenemos la perífrasis modal $<$ poder + infinitivo $>$, es posible introducir el adverbio no entre el auxiliar y el auxiliado. Sin embargo, la perífrasis prograsiva <estar + gerundio > rechaza la interposición de ese mismo elemento (1b).

(1) a. Amancio puede no pagar impuestos.

b. *Amancio está no pagando impuestos.

Podría pensarse que el contraste de (1) se debe a la forma del verbo auxiliado, que es un infinitivo en (1a) y un gerundio en (1b). Sin embargo, datos como los de (2) muestran que hay perífrasis de infinitivo y de gerundio que presentan el comportamiento opuesto a las de (1) y, por tanto, que perífrasis iguales en lo que a su verbo auxiliado se refiere no reaccionan igual ante el mismo material interpuesto:

(2) a. *Echó a no correr.

b. Susana sigue no hablándole a Pedro.

Además, la aceptabilidad depende de la naturaleza del material interpuesto en muchos casos. Así, por ejemplo, mientras que <ir + gerundio $>$ rechaza la interposición de la negación (3b) pero no la del adverbio rápidamente (3a), < deber + infinitivo > presenta la situación opuesta (4).

(3) a. Donald iba rápidamente aprobando un decreto ley tras otro.

b. *Donald iba no admitiendo refugiados. 
(4) a. *Cristina debe rápidamente hacer declaraciones ante el juez.

b. Cristina debe no hacer declaraciones ante el juez.

Finalmente, la misma construcción puede mostrar distribución opuesta con materiales interpuestos en apariencia similares, como se ilustra en (5), donde se admite no, pero se rechaza el adverbio jamás, a pesar de que este también posee valor negativo.
a. *Está no estudiando matemáticas.
b. No está jamás estudiando matemáticas

En este trabajo vamos a intentar establecer un marco general que pueda explicar el material interpuesto en una perífrasis. Dado el gran alcance empírico de este fenómeno, nos centraremos de forma exclusiva en el análisis de las condiciones bajo las cuales el adverbio no puede preceder al verbo auxiliado de una perífrasis. Creemos que el estudio específico de este caso es ilustrativo porque la variabilidad que muestra este adverbio es representativa de otros posibles modificadores, y también porque permite ilustrar la clase de problemas que estos datos tienen para ciertas teorías.

La estructura del trabajo es la siguiente; en el apartado 2 realizaremos una revisión crítica de las principales teorías que podrían explicar el material interpuesto en una perífrasis, mostrando por qué dos de ellas no parecen aptas para enfrentarse a este tipo de datos. En el apartado 3 nos centraremos en el análisis de la interposición negativa en las perífrasis de gerundio a partir de una teoría de dominios; en el apartado 4 extenderemos esta propuesta a las perífrasis de infinitivo. Las conclusiones del trabajo se exponen en el apartado 5.

\section{Tres análisis concebibles sobre el MI}

En este apartado vamos a revisar tres teorías que podrían explicar el material interpuesto (MI, de ahora en adelante) que se admite en una perífrasis: los acercamientos cartográficos, los análisis que se centran en el tamaño sintáctico de las categoría híbridas y las propuestas que dividen la estructura cláusal en dominios ${ }^{1}$.

\footnotetext{
${ }^{1}$ Descartamos de entrada dos propuestas existentes que para el español no dan cuenta de la distribución superficial de los datos. La primera de ellas es la que sostiene que las perífrasis son unidades léxicas (Ackerman, Stump y Webelhuth, 2011). No tendremos en cuenta esta propuesta porque existen datos que constituyen un contraejemplo a esta afirmación.
} 


\subsection{Acercamientos cartográficos}

La primera propuesta que vamos a revisar es la llamada cartográfica (Cinque, 1999).

Las propuestas cartografías postulan la existencia de una secuencia funcional universal y exhaustivamente ordenada. Según esta teoría, la estructura clausal está compuesta por una serie de proyecciones funcionales que están jerárquicamente ordenadas. En (6) ilustramos la propuesta realizada a este respecto por Cinque (1999).

[francamente Mood-speech act [afortunadamente Mood-evaluative [presuntamente Moodevidential [probablemente Mod-epistemic [una vez $\mathrm{T}$ (Past) [entonces $\mathrm{T}$ (Future) [quizás Mood-irrealis [necesariamente Mod-necessity [posiblemente Modpossibility [normalmente Asp-habitual [de nuevo $\quad$ Asp-repetitive(I) $\quad\left[\begin{array}{ll}a & \text { menudo }\end{array}\right.$ Aspfrequentative(I) [intencionadamente Mod-volitional [rápidamente Asp-celerative(I) [ya T(Anterior) [ya no Asp-terminative [todavía Asp-continuative [siempre Asp-perfect [justo Asp-retrospective [pronto Asp-proximative [brevemente Asp-durative [típicamente Asp- generic/progressive [casi Asp-prospective [completamente Asp-SgCompletive(I)

Por una parte, la posibilidad de tener varios verbos axuliados coordinados impide sostener que las perífrasis constituyen una unidad léxica (véase (i)). Por otra, el hecho de que el sujeto pueda aparecer entre el auxiliar y el auxiliado también invalida esa propuesta (véase (ii)).

(i) Coordinación de subconstituyentes

a. Tras haber [pagado las facturas y enviado los recibos], se acostó.

b. Puedes [pasar y sentarte].

c. La empresa iba [adquiriendo casas y demoliéndolas].

(ii) Interpolación de sujeto

a. ¿Cuándo habría mi abuelo aceptado esta clase de bodas?

b. Al tener la princesa que besar a la rana, salió corriendo.

c. Contrataron al joven que tenía Luis visto de la oficina.

La segunda propuesta que vamos a descartar es que el MI admitido sea un efecto prosódico relacionado con la cliticización. Datos como los de (2) muestran que lo que acepta o no una perífrasis como MI no depende de su naturaleza clítica o su poco peso fonológico, puesto que en ese caso la negación debería admitirse o rechazarse en todas las perífrasis. 
[del todo Asp-PlCompletive [bien Voice [rápido / pronto Asp-celerative(II) [de nuevo Asprepetitive(II) [a menudo Asp-frequentative(II) [completamente Asp-SgCompletive(II)

[Cinque, 1999: 106]

Como se observa en (6), la secuencia de proyecciones funcionales contiene posiciones para nociones aspectuales y modales. Así, por ejemplo, existe una proyección funcional dedicada al aspecto progresivo y otra al habitual. Puesto que los auxiliares son, en general, manifestaciones de algunas de estas proyecciones, se situarán en la proyección funcional correspondiente: por ejempo, el auxiliar estar de la perífrasis progresiva ocupará la proyección correspondiente a ese aspecto.

De lo que acabamos de decir se sigue que el MI que acepta una perífrasis dependería de la altura a la que se introdujera el auxiliar en cada caso, y de las proyecciones que dejara disponibles entre su proyección y el verbo. Imaginemos, como se refleja en (7a), que el verbo auxiliar de una perífrasis se relaciona con la noción aspectual o modal correspondiente a la proyección F4 y, por tanto, se sitúa en ella. Si este es el caso, entre el auxiliar y el auxiliado, que se encuentra en el Sv, tenemos tres proyecciones funcionales: F5, F6 y F7. Pues bien, de acuerdo con esta propuesta estructural, en (7a), entre el auxiliar y el auxiliado podríamos introducir el MI que se asocie con las tres proyecciones que aparecen entre los dos verbos, esto es, en F5, F6 y F7. En (7b), puesto que el auxiliar ocupa una posición jerárquicamente inferior, hay menos proyecciones entre este y el auxiliado. En concreto, solo tenemos una proyección entre los dos verbos, F7, y, como consecuencia, solo los elementos que ocupan dicha proyección podrán interponerse entre los dos verbos. A diferencia de lo que sucedía en (7a), en este caso el MI correspondiente a las proyecciones F5 y F6 será rechazado. En (7c) tenemos la situación contraria: el auxiliar ocupa la proyección jerárquicamente superior, por lo que el número de proyecciones que hay entre este verbo y el auxiliado es mayor (F2, F3, F4, F5, F6 y F7), y cualquier elemento relacionado con ellas puede aparecer como MI. De este modo, los acercamientos cartográficos predicen que existe una correlación entre el valor de un auxiliar y la altura del MI que admite la perífrasis: cuanto más alta jerárquicamente sea la proyección en que se situa el auxiliar, más MI admitirá la perífrasis.
a. [F1 [F2 [F3 [F4 auxiliar [F5 [F6 [F7 [Sv auxiliado]]]]]]]]
b. [F1 [F2 [F3 [F4 [F5 [F6 auxiliar [F7 [Sv auxiliado]]]]]]]]
c. [F1 auxiliar [F2 [F3 [F4 [F5 [F6 [F7 [Sv auxiliado]]]]]]]] 
Por tanto, y dado que su secuencia funcional es rígida, las propuestas cartográficas predicen la existencia de relaciones de transitividad en relación con el MI en las perífrasis. La predicción que realizan estos acercamientos es, en concreto, la que recogemos en (8):

(8) Si un elemento MI está jerárquicamente más alto que otro elemento MI, no puede darse el caso de que una perífrasis admita como MI el elemento alto y no el bajo.

Esto se sigue de lo que hemos explicado con respecto a (7). Dado que el MI que admite una perífrasis es aquel que ocupa las proyecciones que se encuentran entre el auxiliar y el auxiliado, no es posible que se rechace la interposición de los elementos de una proyección F7 si se aceptan los de una proyección F6.

Sin embargo, ninguna de estas predicciones se ve corroborada por los datos. En primer lugar, no es evidente que exista correlación alguna entre la altura del auxiliar y la cantidad de MI que admite la perífrasis. Por ejemplo, en la secuencia funcional propuesta por Cinque (6), el orden jerárquico entre las proyecciones de T(anterior), Asp-continuativo y Asp-progresivo es la reflejada en (9).

$$
[\mathrm{T} \text { (anterior) } \ldots[\text { [Asp-continuativo } \ldots[\text { [Asp-progresivo } \ldots]]]
$$

Las proyecciones Asp-continuativo y Asp-progresivo introducen nociones aspectuales expresadas por las perífrasis <continuar + gerundio $>$ y $<$ estar + gerundio $>$, respectivamente, de forma que los auxiliares de estas construcciones se sitúan en dichas proyecciones.
a. $[$ sT(anterior) $\ldots$ [SAsp-continuativo Continuaba ... [sAsp-progresivo ... [Sv queriendo verme $]]$
b. [sT(anterior) ... [sAsp-continuativo ... [sAsp-progresivo Estaba ... [sv corrigiendo los exámenes]]]

Siendo esto así, la aproximación cartográfica predice que el adverbio ya, que se sitúa en $\mathrm{T}$ (anterior), no debería poder aparecer entre el auxiliar y el auxiliado. Esta predicción es correcta en el caso de <continuar + gerundio $>(11 \mathrm{a})$, pero no en el de <estar + gerundio $>$ (11b). 
(11) a. *Continuaba ya queriendo verme.

b. Estaba ya corrigiendo los exámenes.

Tampoco es cierto que existan relaciones de transitividad relativas a la altura en que se sitúa el MI. Si tenemos en cuenta el orden de los elementos de (12) en la jerarquía de Cinque, siempre que ya pueda interponerse en una perífrasis, deberían poder hacerlo brevemente y rápido. Sin embargo, esto no es lo que sucede. Como se muestra en (13), en los tiempos compuestos sucede precisamente lo contrario: el elemento jerárquicamente superior (ya) se acepta (13a), brevemente presenta un menor grado de admisibilidad (13b) y rápidamentel rápido dan lugar a una construcción agramatical $(13 \mathrm{c})^{2}$.

[T(anterior) ya ... [Asp-durativo brevemente ... [Asp-celerativo rápido]]]

(13) a. Había ya empezado su conferencia.

b. ?'Había brevemente departido sobre el Brexit.

c. *Había \{rápidamente / rápido\} contestado el examen.

Consideramos, por todo ello, que no es posible explicar el MI en las perífrasis a partir de los acercamientos cartográficos.

\subsection{Análisis sobre el tamaño de las categorías híbridas}

En esta sección vamos a explorar la posibilidad de dar cuenta del MI a partir de los análisis que se concentran en el tamaño de las categorías híbridas para analizar las perífrasis.

El trasfondo de esta aproximación son las teorías monoclausales sobre la reestructuración (Rizzi, 1978; Wurmbrand, 1998), junto con las observaciones que se remontan a Stowell (1982)

\footnotetext{
${ }^{2}$ Para esquivar estos escollos la teoría cartográfica podría proponer que los adverbios se desplazan, pero si se pueden desplazar fácilmente, entonces toda la jerarquía de Cinque pierde su motivación y sus predicciones.
} 
acerca del tamaño con el que se pueden proyectar los gerundios e infinitivos. La idea fundamental de estas teorías es que los infinitivos y los gerundios pueden poseer más o menos estructura funcional dependiendo del núcleo que los seleccione en cada caso. Los verbos que seleccionan a los infinitivos y gerundios serían, pues, los que determinan si el tamaño de esta categoría verbal sería un Sintagma Complementante (SC), un Sintagma Tiempo (ST) o un Sintagma Verbal (SV), es decir, si el verbo auxiliado en cada caso proyecta más o menos estructura funcional. Por ejemplo, para Wurmbrand (1998), mientras que algunos verbos seleccionan un infinitivo que solo es un SV (14a), otros seleccionan un infinitivo que proyecta como un SC (14b).
a. [Sv $\varnothing[\mathrm{SV}$ intentar [SV leer el libro]]]
b. [Sv preguntar [SC dónde ${ }_{i} \mathrm{C}\left[\mathrm{ST}\left[\mathrm{Sv}\left[\mathrm{SV}\right.\right.\right.$ comer $\left.\left.\left.\left.\left.\mathrm{h}_{\mathrm{i}}\right]\right]\right]\right]\right]$

De acuerdo con estos análisis, la cantidad de MI que contiene una perífrasis dependería de la naturaleza semántica (o tipo semántico) del verbo auxiliado, en la línea de la tipología de infinitivos de Stowell (1982). Lo esperable es que cuanto más estructura proyecte el verbo auxiliado y, por tanto, menos integración tenga con el auxiliar, más MI se permitirá en esa perífrasis. En aquellos casos en que menos estructura haya entre el auxiliar y el auxiliado, menos posibilidades habrá de introducir MI.

La teoría, pues, establece un correlato entre la posibilidad de que haya reestructuración en la perífrasis y admitir menos MI, ya que en ambos casos tendríamos un verbo auxiliado que proyectara el mínimo posible de estructura en el interior de la perífrasis.

Una de las principales pruebas para identificar la reestructuración es la posibilidad de que los clíticos, además de posponerse a la forma no personal, puedan preceder al verbo flexionado. Nótese que esto es lo que sucede en (15), lo que indicaría que estamos ante un caso de reestructuración y que el infinitivo seleccionado por intentar no proyecta prácticamente estructura. En (16), en cambio, el ascenso del clítico no es tan natural, lo que indica que el infinitivo proyecta más estructura que en (15).

(15) a. Intentó saludarla, pero el anfitrión los sentó muy alejados.

b. La intentó saludar, pero el anfitrión los sentó muy alejados.

(16) a. Lleva esperándola dos horas. 
b. ??La lleva esperando dos horas.

Si asumimos las teorías sobre la reestructuración y el tamaño de los infinitivos, lo esperable es que el MI sea más fácilmente aceptado en (16) que en (17), es decir, que haya correlación entre la facilidad con que sucede la reestructuración y la posibilidad de tener MI. Sucede, sin embargo, lo contrario:

(17) a. Intentó no saludarla, pero el anfitrión los sentó uno al lado del otro.

b. La intenté no saludar, pero no me quedó más remedio ${ }^{3}$.

*Lleva no esperándola toda su vida.

\subsection{Una propuesta de dominios}

Vayamos a la última teoría, que será la que defendamos. En trabajos como los de Grimshaw (1991), Ramchand y Svenonius (2014) o Wiltschko (2014) se propone que la estructura clausal se divide en dominios, no en una secuencia rígida de proyecciones. En (19) representamos los dominios y los primitivos semánticos asociados en las propuestas de Ramchand y Svenonius (19a) y Wiltschko (19b).

(19) a. [c proposiciones [T situaciones [v eventos]]]

b. [c enlace [ ${ }_{\mathrm{T}}$ anclaje [Asp perspectiva [v clasificación]]]]

Estos dominios están ordenados, pero, frente a los acercamientos cartográficos, dentro del dominio todo orden o combinación está restringido solamente en función de si produce un resultado interpretable en Forma Lógica y Forma Fonética ${ }^{4}$. En otras palabras, el único orden impuesto por la jerárquia es el correspondiente a los dominios, y el referido al resto de elementos dependerá solo

\footnotetext{
${ }^{3}$ En Ordóñez (2013) se indica que el ascenso de clítico es incompatible con la interposición de la negación. A nuestro juicio, esta generalización no es correcta, puesto que consideramos gramaticales oraciones como la de (17b).

${ }^{4}$ Esta no es, por supuesto, la única diferencia entre los tipos de teorías. Como el lector habrá podido observar, la propuesta de dominios reduce significativamente el número de proyecciones funcionales jerárquicamente ordenadas. No realizaremos aquí una comparación exhaustiva de ambas propuestas. Baste con analizarlas en relación con el fenómeno que estamos estudiando.
} 
de si las configuraciones en que aparezcan son interpretables en For a Lógica (FL) y Forma Fonétic (FF), y no de la supuesta existencia de una plantilla rígida en el estilo de Cinque (1999). Por el mismo motivo, tampoco existen restricciones relativas al dominio en que puede introducirse un elemento.

Si nos atenemos al caso de las perífrasis, estas pertenecen al dominio de las situaciones y de la perspectiva en las propuestas de Ramchand y Svenonius (2014) y Wiltschko (2014), respectivamente (19). Por ello, todo el MI estará o en el dominio de las situaciones o en el límite entre situación y evento. De acuerdo con la teoría de dominios, la posibilidad de que haya MI en una perífrasis no depende del tamaño del auxiliar, ni debe exhibir relaciones de transitividad.

Lo que sí predicen estas teorías, a diferencia de las revisadas antes, son correlaciones entre las nociones semánticas (y fonológicas) que entraña o presupone cada perífrasis y el tipo de MI que admita cada una. La razón es que, como hemos señalado, estas propuestas sostienen que la única condición que restringe el resto de órdenes o combinaciones es su interpretabilidad en FL y FF. Dados los problemas que presentaban las otras dos propuestas, vamos a desarrollar una teoría de dominios, y nos vamos a concentrar, como ya adelantamos en la introducción, en la presencia de no como MI en las perífrasis de gerundio e infinitivo.

\section{Las perífrasis de gerundio y la negación}

En el marco de las teorías que postulan la existencia de dominios defendemos que la posibilidad de interponer una negación entre verbo auxiliar y verbo auxiliado depende específicamente de las condiciones impuestas por el auxiliar, concretamente de si el auxiliar admite en su complemento situaciones no dinámicas. En esta sección enunciaremos esta propuesta para las perífrasis de gerundio.

Los gerundios que forman parte de una perífrasis no admiten siempre la negación (RAE y ASALE, 2009; García Lorente 2014), como muestra el contraste entre (20) y (21) ${ }^{5}$.
a. Comenzó no respondiendo.
b. Empezó no respondiendo.

\footnotetext{
${ }^{5}$ Sobre la anteposición del adverbio no al gerundio no perifrástico véanse Bosque (1980), Sánchez López (1999) y Fernández Lagunilla (2011).
} 
c. Continuó no diciendo la verdad.

d. Terminó no votando en contra.

e. Sigue no queriendo verme.

(21) a. *Anda no estudiando.

b. *Va no corrigiendo exámenes.

c. *Lleva no lloviendo toda la semana.

d. *Viene no lloviendo desde hace una semana.

e. *Está no comiendo.

f. *Se quedó no mirando al paisaje.

Lo que estas perífrasis rechazan no es, al menos en ciertos casos, la interpolación de un elemento negativo, sino la del inductor negativo no. Esto se pone de manifiesto en (22), donde el gerundio puede estar precedido por jamás (22a) pero rechaza la anteposición del adverbio no (22b).
a. No está jamás escribiendo artículos.
b. *Siempre está no escribiendo artículos.

Pasemos a explicar a qué se debe el contraste entre (20) y (21) en el marco de una propuesta de dominios.

\subsection{Propuesta: eventos negativos}

En esencia, nuestra propuesta es que la presencia del inductor negativo no antes del verbo auxiliado se asocia necesariamente a una lectura de evento negativo (Higginbotham, 2000; Arkadiev, 2015). La propiedad fundamental de los eventos negativos que explica estos datos es, según argumentaremos, que los eventos negativos se comportan como eventualidades no dinámicas cuyo inicio se inhibe (Fábregas y González Rodríguez, 2017). Las perífrasis que no admiten el inductor negativo son aquellas que imponen a su complemento la condición de que sea dinámico o esté iniciado.

Por tanto, para presentar nuestra teoría debemos primero hablar brevemente de las propiedades de los eventos negativos, ilustrados a través del infinitivo negado de (23). 
(23) El policía me vio [no parar en el semáforo].

En primer lugar, un evento negativo implica la inhibición de un evento esperado en cierto contexto por parte de un agente; por tanto, un evento negativo es agentivo, pero el papel del agente es el de inhibir el inicio del evento. La diferencia de aceptabilidad en (24) radica en que en el segundo caso no existe un agente externo.

(24) a. Juan llegó a no hervir ni la leche para el desayuno de sus hijos.

b. *La leche llegó a no hervir.

En segundo lugar, el evento negativo es intransformativo, es decir, no dinámico: durante el periodo de vigencia del evento negativo no hay cambios en la situación, precisamente porque el evento negativo implica inhibir que algo suceda; de ahí que se rechacen modificadores necesariamente dinámicos, como poco a poco en (25).

Juan puede no corregir los exámenes (*poco a poco $)^{6}$.

En tercer lugar, pragmáticamente solo tiene sentido mencionar un evento negativo cuando se emite en un contexto en que se presupone o espera que suceda ese evento que se inhibe, de donde se sigue la extrañeza de (26).

(26) \#Puedes no descubrir un nuevo elemento químico.

Finalmente, el evento negativo tiene una duración medible (27a), que corresponde con el tiempo durante el cual el agente inhibe el evento. En esto se diferencia un evento negativo, que sucede durante un periodo de tiempo concreto, de la situación en que simplemente se afirma que no es el caso que suceda un evento, que como tal carece de duración necesariamente (27b).

(27) a. La empresa llegó a no vender casas (durante dos meses).

b. La empresa no llegó a vender casas (*durante dos meses).

\footnotetext{
${ }^{6}$ Debe descartarse la lectura en que poco a poco es el foco de la negación.
} 
Fábregas y González Rodríguez (2017) proponen que la lectura de evento negativo se obtiene cuando la negación está situada internamente al dominio de las eventualidades; así se obtiene la lectura de que en el dominio de las situaciones se instancia el evento que corresponde a la inhibición de la eventualidad. Asumiendo que los auxiliares modales y aspectuales se sitúan en el dominio de las situaciones y no en el de los eventos, la interposición de la negación entre auxiliar y auxiliado corresponde con la situación en que se define un evento negativo.

A efectos de lo que nos interesa en el caso de las perífrasis, la aplicación al análisis del MI es que en (28) tenemos una negación que opera sobre el auxiliar, en el dominio de las situaciones, y por tanto obtenemos la lectura más simple, de evento negado ('no es el caso de que suceda X').
a. No puede pagar impuestos.
b. [s no [st puede $_{\mathrm{i}}\left[\mathrm{smodo} \mathrm{t}_{\mathrm{i}}[\mathrm{sAsp}[\mathrm{sv}\right.$ pagar impuestos]]]]]

En cambio, si el inductor negativo aparece bajo el auxiliar, está operando en el dominio de los eventos y obtenemos la lectura de evento negativo ('es el caso de que no sucede X').
a. Puede no pagar impuestos.
b. $\left[\right.$ st puede $_{i}\left[\right.$ sModo $t_{i}[$ SAsp $[s$ no [sv pagar impuestos]]]]]

\subsection{La naturaleza del evento negativo explica el contraste}

Como se ha visto, el evento negativo tiene propiedades pseudo-estativas, porque es no dinámico y su inicio no implica un cambio, ya que consiste en inhibir el comienzo de una acción: por ejemplo, durante el periodo en que sucede el evento negativo no pagar impuestos no existe ningún cambio en la dimensión definida por ese predicado.

Una vez establecido esto, nótese que las perífrasis de gerundio que rechazan la negación como MI son precisamente aquellas que exigen que el verbo auxiliado sea eventivo e incluso en algunos casos que defina un avance gradual. Ninguna de estas dos condiciones está satisfecha por el evento negativo.

Así, la perífrasis <andar + gerundio>, definida como imperfecta por Martínez-Atienza $\left(2006^{a}\right)$, en palabras de Laca (2006) “exige subintervalos distintos sobre los que se distribuyen los 
eventos" a los que se refiere; se combina preferentemente con actividades (30a), ateliciza los predicados télicos a los que se asocia (30b, c), y rechaza los estados (30d), que dinamiza en la medida en que sean aceptables con ella (30e). Su rechazo de la negación interpuesta es debido, pues, a su rechazo de las eventualidades no dinámicas.
a. Anda buscando un trabajo.
b. Anda escribiendo una novela.
c. Anda recitando el poema.
d. *Anda teniendo dos trabajos a la vez.
e. Anda teniendo cada vez más problemas.

En el caso de <ir + gerundio > también clasificada como imperfecta por Martínez-Atienza (2006b), se dice de ella que "aporta una estructura temporal incremental" (Laca, 2006), por lo que también exige auxiliados dinámicos, y concretamente aquellos que admitan una interpretación de avance gradual.

(31) a. Iba leyendo una novela.

b. *Iba encontrando un mueble de su gusto.

c. Iba llegando cada vez más mercancia.

d. *Iba teniendo dos casas.

Ya que los eventos negativos no son dinámicos ni admiten la interpretación mencionada, esta perífrasis rechaza la negación interpuesta.

En cuanto a la perífrasis progresiva <estar + gerundio > es bien conocido que también exige un auxiliado dinámico, por lo que de nuevo rechaza la negación interpuesta por motivos idénticos a los otros casos.

(32) a. *Está teniendo dos casas.

b. Está corriendo por el parque.

c. Está escribiendo una novela.

d. Está llegando a casa. 
Exactamente lo mismo sucede con la perífrasis continuativa <llevar + gerundio > (33a), la resultativa $<$ quedarse + gerundio $>$ (33b) y la también continuativa <venir + gerundio $>(33 \mathrm{c})$.

(33) a. *Lleva toda la vida teniendo dos trabajos.

b. *Se quedó teniendo dos trabajos.

c. *Viene teniendo dos trabajos desde que lo conozco.

En cambio, todas las perífrasis de gerundio que admiten la negación como MI también admiten predicados estativos como auxiliados; su aceptación de la negación como MI es un reflejo de que aceptan complementos no dinámicos. Esto aplica a las perífrasis discursivas <comenzar + gerundio > (34), <empezar + gerundio > (35) y <terminar + gerundio > (36), así como a <continuar + gerundio $>$ (37) y <seguir + gerundio $>$ (38).

(34) a. Comenzó no respondiendo.

b. Comenzó teniendo un solo trabajo y ahora tiene tres.

(35) a. Empezó no respondiendo.

b. Empezó sabiendo solo inglés y ahora habla seis idiomas.

(36) a. Terminó no votando en contra.

b. Terminó teniendo dos trabajos porque no podía pagar el alquiler.

(37) a. Continuó no diciendo la verdad.

b. Continuó teniendo dos trabajos hasta que no pudo más.

(38) a. Sigue no queriendo verme.

b. Sigue teniendo dos trabajos.

\section{Las perífrasis de infinitivo}

La conclusión de la sección anterior puede resumirse como sigue: la interposición del inductor negativo entre auxiliar y auxiliado fuerza una lectura de evento negativo, por lo que las perífrasis que imponen alguna propiedad incompatible con esta lectura rechazan dicha interposición. En esta sección extenderemos el análisis a las perífrasis de infinitivo. 
Comenzando por las perífrasis temporoaspectuales de infinitivo, y concretamente por las perífrasis de lectura incoativa, comprobamos que solo algunas de ellas son compatibles con la negación (39). La mayoría no lo son (40).

(39) a. Empezó a no pagar impuestos.

b. Comenzó a no pagar impuestos.

(40) a. *Arrancó a no hablar.

b. *Echó a no correr.

c. *Se echó a no llorar.

d. *Se lió a no hablar.

e. *Rompió a no gritar.

La explicación de esta incompatibilidad puede relacionarse directamente con las condiciones de un evento negativo. Las perífrasis de (40) son aquellas que imponen al verbo auxiliado la condición de que debe expresar acciones dinámicas que impliquen cambios internos al desarrollo del evento, como muestra la agramaticalidad de (41), incluso para verbos no estativos que no implican cambios internos, como dormir o esperar.

(41) a. *Arrancó a dormir.

b. *Echó a estar sentado.

c. *Se echó a pensar.

d. *Se lió a esperar.

e. *Rompió a escuchar.

Es interesante el caso de <ponerse $a+$ infinitivo $>$. En este caso la perífrasis no impone un requisito de que la acción que se inicia deba tener un desarrollo dinámico interno que marque el inicio:

(42) a. Se puso a pensar en el tema.

b. Se puso a dormir sin esperar a nadie. 
En principio, la combinatoria con la negación interpuesta está limitada, pero en el Corpus del Español de Mark Davies se documentan casos en los que la propiedad crucial parece ser que el evento negativo se inicia voluntariamente y de forma consciente por el sujeto:

a. Porque yo no puedo, si me pongo a no pensar nada, ya estoy pensando en no pensar. b. Creo que nos deberíamos poner a no vender las patentes de motores eficientes que no usan hidrocarburos.

Esto encaja bien con el hecho de que esta perífrasis sea incompatible con eventos accidentales o aquellos que, controlados o no, no impliquen la participación consciente del sujeto:
a. *Me puse a caer por las escaleras.
b. *Me puse a descubrir nuevos manuscritos medievales.
c. *Me puse a toparme con mis colegas por la ciudad.
d. *Me puse a recibir cartas.

La perífrasis prospectiva <ir $a+$ infinitivo $>$ admite la negación interpuesta, como se espera del hecho de que acepte predicados estativos.
a. Voy a no hablarle más.
b. Voy a estar en casa.

En cuanto a la negación como MI en el caso de las perífrasis modales, nuestra predicción es que debería admitirse en todos los casos, ya que todas las perífrasis modales admiten complementos estativos. Esta predicción no produce problemas en general, ya que se documentan casos en todas ellas:

(46) a. Puedes no pagar impuestos.

b. Debes no enfrentarte a tus colegas.

c. Alguien dígale al gringo que por dignidad debería de no aceptar las convocatorias.

d. Sólo he de no salir ningún día. 
e. Hay que no tener alma para que no te guste Natsume.

f. Lo único importante es que Castro tenía que no saberlo.

Podríamos dejar esto aquí como una predicción que se cumple, pero queremos hacer explícito el hecho de que los hablantes consultados a menudo tienen dudas o encuentran algo más marcada la interposición de la negación con algunas de estas perífrasis; concretamente, hablantes nativos del español -incluyendo a los autores de este artículo- encuentran que (46c-f) son algo más marcadas que las dos primeras. Coincide que todas estas son perífrasis modales en las que entre auxiliar y auxiliado hay un elemento más, y sobre todo cuando el elemento intermedio es que. Los autores de este artículo coinciden en observar que (47b) es más natural que (47c), y (47a) lo es más que ambas.
a. Se debe no comer azúcar.
b. 'Se ha de no comer azúcar.
c. 'Hay que no comer azúcar.

Pese a que esto es algo que habrá que dejar para investigación posterior, podemos hacer alguna sugerencia sobre qué puede estar sucediendo aquí. Tanto de como que son elementos fonológicamente deficientes que pueden introducir oraciones subordinadas completivas en español:
a. Dijo de ir al cine.
b. Dijo que fuéramos al cine.

Nuestra propuesta es que la menor aceptabilidad de (47b) y (47c) sí es en este caso un efecto prosódico, concretamente un efecto de que, como elementos fonológicamente defectivos dentro de la perífrasis, de y que quieren tener adyacencia estricta al verbo auxiliado, y la presencia de la negación lo impide. No obstante, como decimos, esta aceptabilidad reducida merece investigaciones más profundas, con uso de métodos experimentales.

\section{Conclusiones}


Cerremos este trabajo exponiendo sus conclusiones más importantes.

La principal conclusión es que una teoría de dominios, a diferencia de la propuesta cartográfica y de la que está basada en el tamaño estructural de los verbos auxiliados, evita las predicciones incorrectas que hacen las otras teorías con respecto al MI que admite una perífrasis. Creemos haber sido capaces de argumentar a favor de un análisis en que la aceptabilidad del inductor negativo como MI en el interior de una perífrasis depende de las condiciones semánticas que cada auxiliar impone a su complemento. Ya que la negación da lugar en esa posición a una lectura de evento negativo, qué perífrasis admiten negación como MI depende de qué auxiliares admiten complementos con las propiedades de esta clase de eventos.

Los eventos negativos se caracterizan, entre otras cosas, por su falta de dinamicidad. Las perífrasis que no admiten la negación como MI son precisamente aquellas que rechazan los predicados no dinámicos como complementos; a la inversa, aquellas perífrasis de gerundio que admiten predicados estativos aceptan la negación.

En el caso de las perífrasis de infinitivo, la posibilidad de introducir la negación como MI también obedece a los requisitos aspectuales que impone. Así, rechazan la negación aquellas perífrasis que imponen a su complemento la condición de que exprese un evento dinámico cuyo inicio sea el comienzo de un cambio o de un desarrollo posterior, algo que es incompatible con la semántica de los eventos negativos, en los que el sujeto inhibe la acción que se esperaba que tuviera lugar.

Con respecto a las perífrasis modales, en todas ellas se documentan casos con negación como MI, tal y como esperamos, pero también es cierto que los juicios de los hablantes son más variables en aquellas donde el verbo auxiliar va seguido de que y de. A este respecto hemos sugerido provisionalmente que esos elementos son clíticos que deben estar en adyacencia estricta con el verbo auxiliado.

En conclusión, este análisis del MI, pese a estar restringido a uno solo de los subcasos posibles, ha proporcionado argumentos a favor de un tratamiento composicional donde el material interpuesto no puede ser predicho ciegamente a partir de propiedades jerárquicas o el tamaño funcional de un elemento, sino que ha de tener en cuenta las condiciones semánticas que aporta el modificador y su aceptabilidad en la perífrasis. Aunque son muchos los casos a los que este tipo de análisis debe ser extendido para poder afirmar que un tratamiento mediante dominios es el que mejor equipado está para enfrentarse a este problema, esperamos al menos haber argumentado 
convicentemente en este trabajo que los dominios son el mejor modelo para tratar los casos de inductores negativos internos a una perífrasis.

\section{Bibliografía citada}

ACKERMAN, Gregory T., Farrell StUMP y Gert WeBELHUTH, 2011: "Lexicalism, periphrasis and implicative morphology” en Bob BORSLEY y Kjersti BORJARS (eds.): Non-transformational theories of grammar, Oxford, New York: Blackwell, 325-358.

ARKADIEV, Peter, 2015: "Negative events: evidence from Lithuanian" en Peter ARKADIEV, Ivan

Kapitonov, Yury LANDER, Ekaterina RaKhilina y Sergei TATEvosov (eds.): Donum semanticum: Opera linguistica et logica in honorem Barbarae Partee a discipulis amicisque Rossicis oblate, Mocú: LRC, 7-20.

BoSQUE, Ignacio, 1980: “La preposición sin”. Lingüística Hispánica II, 71-85..

CINQUE, Guglielmo, 1999: Adverbs and functional heads: A cross-linguistic perspective, New York: Oxford University Press.

FÁBREGAS, Antonio y Raquel GONZÁleZ RoDRíGUEZ, 2017: “Building negative events", manuscrito no publicado..

FERNÁNDEZ DE CASTRO, Félix, 1999: Las perífrasis verbales en el español actual, Madrid: Gredos.

FERNÁNDEZ LAGUNILlA, Marina, 2011: "Restricciones de la negación con el gerundio adjunto modal" en Manuel LeONETTI, M. Victoria EsCANDELl y Cristina SÁNCHEZ (eds.): 60 problemas de gramática, Madrid: Akal, 247-252.

GARCÍA FERNÁNDEZ, Luis (dir.), 2006: Diccionario de perífrasis verbales, Madrid: Gredos.

GARCía GonZÁLEZ, Javier, 1992: Perífrasis verbales, Madrid: Sociedad General Española de Librería.

GARCÍA LORENTE, María José, 2014: “No escrito”, “no escribiendo”, “sin escribir”. Formas de negar el gerundio y el participio en español. Trabajo de Fin de Máster, Universidad Complutense de Madrid.

GÓMEZ TORREGO, Leonardo, 1999: “Los verbos auxiliares. Las perífrasis de infinitivo” en Ignacio BosQue y Violeta DEMONTE (eds.): Gramática descriptiva de la lengua española, Madrid: Espasa Calpe, 3323-3389.

GRIMSHAW, Jane, 1991: “Extended projections”, manuscrito no publicado. 
HigGinbOTHAM, James, 2000: “On events in linguistics semantics” en J. HigGinbotham, Fabio PIANESI y Achielle C. VARZI (eds.): Speaking of events, Oxford: Oxford University Press, 49-79.

LACA, Brenda, 2002: "Spanish aspectual periphrases: ordering constraints and the distinction between situation and viewpoint aspect" en Javier GUTIÉRREZ REXACH (ed.): From words to discourse, Oxford: Elsevier, 61-95.

LACA, Brenda, 2006: "Pluralidad y aspecto verbal en español”, Revista Española de Lingüística $36,7-41$.

MARTíneZ-AtiEnZA, María, 2006a: “<Andar + gerundio>” en Luis GARCÍA FERnÁndeZ (ed.): Diccionario de perífrasis verbales, Madrid: Gredos, 268-272.

MARTíneZ-ATIEnZA, María, 2006b: “<Ir + gerundio>” en Luis GARCÍA FernáNDEZ (ed.): Diccionario de perífrasis verbales, Madrid: Gredos, 172-175

ORDÓÑEZ, Franciso, 2013: "El movimiento de los clíticos” en José M. a BrUCART y Ángel J. GALLEGO (eds.): El movimiento de constituyentes, Madrid: Virsor, 107-122.

REAl ACADEMIA ESPAÑOLA y ASOCIACIÓN DE ACADEMIAS DE LA LENGUA ESPAÑOLA, 2009: Nueva gramática de la lengua española, Madrid: Espasa Calpe.

RAMCHAND, Gillian y Peter SvenONIUS, 2014: "Deriving the functional hierarchy" Language Sciences 46, 152-174.

RIZZI, Luigi, 1978: “A restructuring rule in Italian syntax” en Samuel Jay KEYSER (ed.): Recent transformational studies in European languages, Cambridge, Ma.: MIT Press, 113-158.

RoCA PONS, José, 1958: Estudios sobre perífrasis verbales del español, anejo XVII del Boletín de la Real Academia Española, Madrid.

SÁNCHEZ LóPEZ, Cristina. 1999: "La negación” en Ignacio BoSQUE y Violeta DEMONTE (eds.): Gramática descriptiva de la lengua española, Madrid: Espasa Calpe, 2561-2634.

SQUARTINI, Mario, 1998: Verbal periphrases in Romance, Berlin: Mouton de Gruyter.

STOWELL, Tim, 1982: “The tense of infinitives”, Linguistic Inquiry 13(3), 561-570.

WiltschKo, Martina, 2014: The universal structure of categories, Cambridge: Cambridge University Press.

Wurmbrand, Susi, 1998: "Heads or phrases? Particles in particular" en W. KEHREIN y R. WIESE (eds.): Phonology and morphology of the Germanic languages, Tübingen: Niemeyer, 267295. 
YLLERA, Alicia, 1999: "Las perífrasis verbales de gerundio y participio” en Ignacio BosQUE y Violeta DEMONTE (eds.): Gramática descriptiva de la lengua española, Madrid: Espasa Calpe, 3391-3441. 